\title{
Quasi-Amicable Numbers
}

\author{
By Peter Hagis, Jr. and Graham Lord
}

\begin{abstract}
If $m=\sigma(n)-n-1$ and $n=\sigma(m)-m-1$, the integers $m$ and $n$ are said to be quasi-amicable numbers. This paper is devoted to a study of such numbers.
\end{abstract}

Let $\sigma(N)$ denote the sum of the positive divisors of the integer $N($ where $N>1$ ), and let

$$
L(N)=\sigma(N)-N-1
$$

so that $L(N)$ is the sum of the "nontrivial" divisors of $N$. A $q t$-cycle (" $q$ " for quasi) is a $t$-tuple of distinct positive integers $\left(m_{1}, m_{2}, \ldots, m_{t}\right)$ such that $m_{i}=L\left(m_{i-1}\right)$ for $i \neq 1$ and $m_{1}=L\left(m_{t}\right)$. A $q 1$-cycle is usually called a quasi-perfect number; and we shall call $q 2$-cycles quasi-amicable numbers. (In both [2] and [5] $q 2$-cycles are referred to as "reduced" amicable pairs. Garcia, however, calls them "números casi amigos" (see the editorial note in [5]).) No quasi-perfect numbers have been found as yet; and if one exists, it exceeds $10^{20}$ (see [1]). They have been studied by Cattaneo [3], Abbott-Aull-Brown-Suryanarayana [1], and Jerrard-Temperley [4].

In [5] Lal and Forbes list the nine quasi-amicable pairs with smallest member less than $10^{5}$. Beck and Najar [2] have continued the search as far as $10^{6}$ and found six more quasi-amicable pairs. Using the CDC 6400 at the Temple University Computing Center, a search was made for all quasi-amicable pairs with smallest member less than $10^{7}$. Forty-six pairs were found including two (526575-544784 and 573560817479 ) with smallest member between $10^{5}$ and $10^{6}$ which apparently were missed by Beck and Najar [2]. These are listed at the end of this paper. (For the sake of convenience and completeness the pairs given in [2] and [5] are included here.)

It will be noticed that each pair in our list is of opposite parity, leading us to inquire: Are there any quasi-amicable pairs of the same parity? Now, the positive integers $m$ and $n$ are quasi-amicable if and only if $L(m)=n$ and $L(n)=m$ so that from (1), we have

$$
\sigma(m)=\sigma(n)=m+n+1
$$

Therefore, since $\sigma(N)$ is odd if and only if $N=S^{2}$ or $N=2 S^{2}$, we see that a necessary condition for $m$ and $n$ to be quasi-amicable numbers of the same parity is that each be a square or twice a square. Making use of this fact a search was made for quasi-amicable pairs of the same parity in the range $\left[10^{7}, 10^{10}\right]$. None was found so

Received March 8, 1976; revised October 18, 1976.

AMS (MOS) subject classifications (1970). Primary $10 \mathrm{~A} 20$. 
that we have:

Proposition 1. If $m<n$ and $(m, n)$ is a quasi-amicable pair having the same parity, then $m>10^{10}$.

If $m$ and $n$ are relatively prime quasi-amicable numbers then, using (2), we have $\sigma(m n) / m n=\sigma(m) \sigma(n) / m n=(m+n+1)^{2} / m n>(m+n)^{2} / m n=2+m / n+n / m$ $>4$. Since, if $p$ is a prime, $\sigma\left(p^{\alpha}\right) / p^{\alpha}<p /(p-1)$ and since $x /(x-1)$ is a decreasing function, it follows that if $m n$ has less than four prime factors then $\sigma(m n) / m n<$ $(2 / 1)(3 / 2)(5 / 4)<4$. If $m n$ is odd and $m n$ has fewer than twenty-one prime factors, then

$$
\sigma(m n) / m n<(3 / 2)(5 / 4)(7 / 6) \cdots(71 / 70)(73 / 72)<4 \text {. }
$$

We have proved:

Proposition 2. Let $m$ and $n$ be relatively prime quasi-amicable numbers. Then $m n$ has at least four different prime factors. If, also, $m$ and $n$ have the same parity (so that $\mathrm{mn}$ is odd), $\mathrm{mn}$ has at least twenty-one different prime factors.

Now, if $(m, n)=1$ and $m n$ is odd then, as noted earlier, $m$ and $n$ are squares so that from Proposition 2 we have $m n \geqslant(3 \cdot 5 \cdot 7 \cdot \cdots 73 \cdot 79)^{2}>25 \cdot 10^{59}$. If $n>m$ and $n<2.5 m$, then $2.5 m^{2}>m n>25 \cdot 10^{59}$ and $m>10^{30}$. If $n>2.5 m$, then $\sigma(m) / m=(m+n+1) / m>3.5$. $m$ has at least thirteen prime factors since $(3 / 2)(5 / 4) \cdots(37 / 36)(41 / 40)<3.5$; and $m>(3 \cdot 5 \cdot 7 \cdots 41 \cdot 43)^{2}>10^{30}$. We have established:

PROPOSITION 3. If $m$ and $n$ are relatively prime quasi-amicable numbers of the same parity, then $m$ and $n$ each exceeds $10^{30}$.

We note that no number in our list of quasi-amicable numbers is a prime power. If $p^{\alpha}$ and $n$ are quasi-amicable numbers then, of course, $\alpha>1$. If $p=2$, then $\dot{\sigma}(n)$ $\left(=\sigma\left(p^{\alpha}\right)\right)$ is odd so that $n$ is of the form $S^{2}$ or $2 S^{2}$. From (2) $2^{\alpha+1}-1=2^{\alpha}+$ $n+1$ so that $n=2\left(2^{\alpha-1}-1\right)$. Therefore, $S^{2}=2^{\alpha-1}-1$. But this is impossible since $S^{2} \equiv 1(\bmod 8)$ and $2^{\alpha-1}-1 \equiv-1(\bmod 8)$. (For $\alpha>3$ since neither $2^{2}$ nor $2^{3}$ is a member of a quasi-amicable pair.) If $p$ is odd and $2 \mid \alpha$, then $\sigma(n)$ and $n$ are both odd and $n=S^{2}$. From (2) $1+p+\cdots+p^{\alpha}=p^{\alpha}+n+1$ so that $n=$ $p\left(1+p+\cdots+p^{\alpha-2}\right)$. Thus, $p \| n$ which contradicts $n=S^{2}$. Since we now know that $p$ and $\alpha$ are both odd, it follows that $\sigma\left(p^{\alpha}\right)$ and $n$ are both even. If $\alpha=3, n=$ $p(1+p)$ so that

$$
\begin{aligned}
\sigma(n) & =(1+p) \sigma(1+p)<(1+p)(1+2+3+\cdots+(p+1)) \\
& =(1+p)(1+p)(2+p) / 2 .
\end{aligned}
$$

But $\sigma(n)=\sigma\left(p^{3}\right)=(1+p)\left(1+p^{2}\right)$. It follows that $2+2 p^{2}<2+3 p+p^{2}$ so that $p<3$. This contradiction completes the proof of

Proposition 4. If $\left(p^{\alpha}, n\right)$ is a quasi-amicable pair, then $p$ is an odd prime, $\alpha$ is an odd number greater than 3 , and $n$ is even.

COROLlARY 4.1. It is not possible for both members of a quasi-amicable pair to be prime powers. 


\section{Quasi-Amicable Pairs}

$$
\begin{aligned}
& 48=2^{4} \cdot 3 \\
& 140=2^{2} \cdot 5 \cdot 7 \\
& 1050=2 \cdot 3 \cdot 5^{2} \cdot 7 \\
& 1575=3^{2} \cdot 5^{2} \cdot 7 \\
& 2024=2^{3} \cdot 11 \cdot 23 \\
& 5775=3 \cdot 5^{2} \cdot 7 \cdot 11 \\
& 8892=2^{2} \cdot 3^{2} \cdot 13 \cdot 19 \\
& 9504=2^{5} \cdot 3^{3} \cdot 11 \\
& 62744=2^{3} \cdot 11 \cdot 23 \cdot 31 \\
& 186615=3^{2} \cdot 5 \cdot 11 \cdot 13 \cdot 29 \\
& 196664=2^{3} \cdot 13 \cdot 31 \cdot 61 \\
& 199760=2^{4} \cdot 5 \cdot 11 \cdot 227 \\
& 266000=2^{4} \cdot 5^{3} \cdot 7 \cdot 19 \\
& 312620=2^{2} \cdot 5 \cdot 7^{2} \cdot 11 \cdot 29 \\
& 526575=3 \cdot 5^{2} \cdot 7 \cdot 17 \cdot 59 \\
& 573560=2^{3} \cdot 5 \cdot 13 \cdot 1103 \\
& 587460=2^{2} \cdot 3 \cdot 5 \cdot 9791 \\
& 1000824=2^{3} \cdot 3 \cdot 11 \cdot 17 \cdot 223 \\
& 1081184=2^{5} \cdot 13 \cdot 23 \cdot 113 \\
& 1139144=2^{3} \cdot 23 \cdot 41 \cdot 151 \\
& 1140020=2^{2} \cdot 5 \cdot 7 \cdot 17 \cdot 479 \\
& 1173704=2^{3} \cdot 7 \cdot 20959 \\
& 1208504=2^{3} \cdot 11 \cdot 31 \cdot 443 \\
& 1233056=2^{5} \cdot 11 \cdot 31 \cdot 113 \\
& 1236536=2^{3} \cdot 7 \cdot 71 \cdot 311 \\
& 1279950=2 \cdot 3 \cdot 5^{2} \cdot 7 \cdot 23 \cdot 53 \\
& 1921185=3^{3} \cdot 5 \cdot 7 \cdot 19 \cdot 107 \\
& 2036420=2^{2} \cdot 5 \cdot 19 \cdot 23 \cdot 233 \\
& 2102750=2 \cdot 5^{3} \cdot 13 \cdot 647 \\
& 2140215=3 \cdot 5 \cdot 7 \cdot 11 \cdot 17 \cdot 109 \\
& 2171240=2^{3} \cdot 5 \cdot 17 \cdot 31 \cdot 103 \\
& 2198504=2^{3} \cdot 7 \cdot 11 \cdot 43 \cdot 83 \\
& 2312024=2^{3} \cdot 11 \cdot 13 \cdot 43 \cdot 47 \\
& 2580864=2^{7} \cdot 3 \cdot 11 \cdot 13 \cdot 47 \\
& 2958500=2^{2} \cdot 5^{3} \cdot 61 \cdot 97 \\
& 4012184=2^{3} \cdot 11 \cdot 127 \cdot 359 \\
& 4311024=2^{4} \cdot 3 \cdot 19 \cdot 29 \cdot 163 \\
& 75=3 \cdot 5^{2} \\
& 195=3 \cdot 5 \cdot 13 \\
& 1925=5^{2} \cdot 7 \cdot 11 \\
& 1638=2^{4} \cdot 103 \\
& 2295=3^{3} \cdot 5 \cdot 17 \\
& 6128=2^{4} \cdot 383 \\
& 16587=3^{2} \cdot 19 \cdot 97 \\
& 20735=5 \cdot 11 \cdot 13 \cdot 29 \\
& 75495=3 \cdot 5 \cdot 7 \cdot 719 \\
& 206504=2^{3} \cdot 83 \cdot 311 \\
& 219975=3 \cdot 5^{2} \cdot 7 \cdot 419 \\
& 309135=3 \cdot 5 \cdot 37 \cdot 557 \\
& 507759=3 \cdot 7 \cdot 24179 \\
& 549219=3 \cdot 11^{2} \cdot 17 \cdot 89 \\
& 544784=2^{4} \cdot 79 \cdot 431 \\
& 817479=3^{3} \cdot 13 \cdot 17 \cdot 137 \\
& 1057595=5 \cdot 7 \cdot 11 \cdot 41 \cdot 67 \\
& 1902215=5 \cdot 7 \cdot 17 \cdot 23 \cdot 139 \\
& 1331967=3 \cdot 7^{2} \cdot 13 \cdot 17 \cdot 41 \\
& 1159095=3 \cdot 5 \cdot 7^{2} \cdot 19 \cdot 83 \\
& 1763019=3^{3} \cdot 17 \cdot 23 \cdot 167 \\
& 1341495=3^{3} \cdot 5 \cdot 19 \cdot 523 \\
& 1348935=3 \cdot 5 \cdot 7 \cdot 29 \cdot 443 \\
& 1524831=3 \cdot 7^{2} \cdot 11 \cdot 23 \cdot 41 \\
& 1459143=3^{2} \cdot 7 \cdot 19 \cdot 23 \cdot 53 \\
& 2576945=5 \cdot 7 \cdot 17 \cdot 61 \cdot 71 \\
& 2226014=2 \cdot 7 \cdot 17 \cdot 47 \cdot 199 \\
& 2681019=3^{5} \cdot 11 \cdot 17 \cdot 59 \\
& 2142945=3^{2} \cdot 5 \cdot 7 \cdot 6803 \\
& 2421704=2^{3} \cdot 263 \cdot 1151 \\
& 3220119=3^{2} \cdot 7 \cdot 79 \cdot 647 \\
& 3123735=3 \cdot 5 \cdot 29 \cdot 43 \cdot 167 \\
& 3010215=3 \cdot 5 \cdot 13 \cdot 43 \cdot 359 \\
& 5644415=5 \cdot 7 \cdot 29 \cdot 67 \cdot 83 \\
& 3676491=3^{2} \cdot 7 \cdot 13 \cdot 67^{2} \\
& 4282215=3 \cdot 5 \cdot 7 \cdot 17 \cdot 2399 \\
& 7890575=5^{2} \cdot 7 \cdot 11 \cdot 4099
\end{aligned}
$$




$$
\begin{array}{ll}
5088650=2 \cdot 5^{2} \cdot 7^{2} \cdot 31 \cdot 67 & 6446325=3 \cdot 5^{2} \cdot 23 \cdot 37 \cdot 101 \\
5416820=2^{3} \cdot 5 \cdot 43 \cdot 47 \cdot 67 & 7509159=3^{3} \cdot 7 \cdot 67 \cdot 593 \\
6081680=2^{4} \cdot 5 \cdot 11 \cdot 6911 & 9345903=3 \cdot 7 \cdot 17 \cdot 47 \cdot 557 \\
6618080=2^{5} \cdot 5 \cdot 7 \cdot 19 \cdot 311 & 12251679=3 \cdot 11 \cdot 17 \cdot 21839 \\
7460004=2^{2} \cdot 3 \cdot 23 \cdot 151 \cdot 179 & 10925915=5 \cdot 7 \cdot 11 \cdot 13 \cdot 37 \cdot 59 \\
7875450=2 \cdot 3^{2} \cdot 5^{2} \cdot 11 \cdot 37 \cdot 43 & 16381925=5^{2} \cdot 7^{2} \cdot 43 \cdot 311 \\
8713880=2^{3} \cdot 5 \cdot 7 \cdot 31121 & 13693959=3^{2} \cdot 17 \cdot 37 \cdot 41 \cdot 59 \\
8829792=2^{5} \cdot 3^{2} \cdot 23 \cdot 31 \cdot 43 & 18845855=5 \cdot 7 \cdot 23 \cdot 41 \cdot 571 \\
9247095=3^{3} \cdot 5 \cdot 11 \cdot 13 \cdot 479 & 10106504=2^{3} \cdot 47 \cdot 26879
\end{array}
$$

Acknowledgement. The authors were informed almost simultaneously and inde: pendently by the referee and Professor R. Najar of the work of Lal and Forbes [5] on "quasi-aliquot" sequences and quasi-amicable numbers. An earlier version of the present paper contained many of the results to be found in [5], and we are grateful for being informed of this duplication of effort.

Department of Mathematics

Temple University

Philadelphia, Pennsylvania 19122

Département de Mathématiques (Actuariat)

Université Laval

Québec, P.Q. G1 K 7P4, Canada

1. H. L. ABBOTT, C. E. AULL, E. BROWN \& D. SURYANARAYANA, "Quasiperfect numbers," Acta Arith., v. 22, 1973, pp. 439-447. MR 47 \#4915. appear.)

2. W. E. BECK \& R. M. NAJAR, "More reduced amicable pairs," Fibonacci Quart. (To

3. P. CATtANEO, "Sui numeri quasiperfetti," Boll. Un. Mat. Ital., v. 6, 1951, pp. 59-62.

4. R. P. JERRARD \& N. TEMPERLEY, "Almost perfect numbers," Math. Mag., v. 46, 1973, pp. 84-87. MR 51 \#12686.

5. M. LAL \& A. FORBES, “A note on Chowla's function," Math. Comp., v. 25, 1971, pp. 923-925. MR 45 \#6737. 